\title{
Prominent Eustachian Valve in Newborns: A Report of Four Cases
}

\author{
Ashraf Gad, MD ${ }^{1}$ Javed Mannan, MD² Manoj Chhabra, MD ${ }^{1} \quad$ Xi Xiao Yang Zhang, MS ${ }^{1}$ \\ Pramod Narula, MD ${ }^{1}$ Danthanh Hoang, MD ${ }^{1}$
}

${ }^{1}$ Department of Pediatrics, NY Methodist Hospital, Brooklyn, New York

2 Department of Pediatrics, University of Rochester Medical Center, Rochester, New York

Address for correspondence Ashraf Gad, MD, Department of Pediatrics, NY Methodist Hospital, 506 6th Street, Brooklyn, NY 11215 (e-mail: imamgad@hotmail.com).

Am J Perinatol Rep 2016;6:e33-e37.

\author{
Abstract \\ Keywords \\ - Eustachian valve \\ - newborn \\ - cyanosis \\ - echocardiogram \\ - infant
}

The Eustachian valve (EV) is an embryological remnant of the inferior vena cava that during fetal life helps divert oxygenated blood from the IVC toward the foramen ovale to escape the pulmonary circulation. This remnant usually regresses after birth and is considered a benign finding in the majority of cases. However, EV can lead to complications in the neonatal period or later in life. In this short case series, we present four newborn infants with prominent $\mathrm{EV}$ who were symptomatic after birth and required admission to the neonatal intensive care unit.
The Eustachian valve (EV) is located in the superior portion of the inferior vena cava (IVC) and protrudes into the right atrial cavity. It is considered to be a functional valve in the fetus that helps direct oxygenated blood from the IVC toward the foramen ovale, thereby bypassing the pulmonary circulation. ${ }^{1}$ After birth and upon closure of the foramen ovale, the valve has no particular function and tends to regress. There is a large variability in the nature and characteristics of the $\mathrm{EV}$ in regards to shape, size, thickness, texture, and length. ${ }^{1,2}$ Historically, a prominent $\mathrm{EV}$ is essentially benign in nature and requires no treatment. However, it can cause significant intracardiac shunting of deoxygenated blood from right-to-left atrium. In this report, we present clinical case vignettes of four newborn infants who were admitted to the neonatal intensive care unit (NICU) with cyanosis and oxygen $\left(\mathrm{O}_{2}\right)$ desaturation. They were all found to have prominent $\mathrm{EV}$ with significant intracardiac right-to-left shunting.

\section{Case 1}

A $3.15 \mathrm{~kg}$ term female newborn was admitted to the NICU due to cyanosis and stridor while crying. She was born via vacuum-assisted vaginal delivery. Her mother had gestational diabetes treated with glyburide. Prolonged fetal decelerations were recorded during external fetal heart rate monitoring. At birth, the baby required positive pressure ventilation for 60 seconds and was assigned Apgar scores of 5 and 8 at 1 and 5 minutes, respectively. At 26 hours of life, the baby was observed to turn pale with crying. The baby was placed on a pulse oximeter, and with crying, would desaturate to the low 70s. Saturations would return to the high 90s immediately upon the administration of $2 \mathrm{~L}$ of free flow $\mathrm{O}_{2}$. When not agitated, her saturations on room air were 95 to $100 \%$. On examination, she was noted to have circumoral cyanosis, as well as stridor with suprasternal retractions apparent only when she was crying. Mild micrognathia was also noted. Airway and lungs were clear on chest X-ray (CXR) and without consolidations, atelectasis, pneumothorax, or pleural effusion. Echocardiography (Echo) showed normal anatomy with good left ventricular (LV) function. A prominent EV was visible, causing blood to shunt right-to-left from the IVC to the left atrium (LA). A patent foramen ovale (PFO) was present with intermittent right-to-left shunting mainly in diastole (-Fig. 1). Echo study also ruled out the Chiari network (CN) and cor triatriatum dexter (CTD). No other anomaly seen and the coronary sinus ostium looked normal. No other received

March 24, 2015

accepted after revision

August 21, 2015

published online

October 28, 2015
DOI http://dx.doi.org/

10.1055/s-0035-1565920. ISSN 2157-7005.
Copyright $\odot 2016$ by Thieme Medical Publishers, Inc., 333 Seventh Avenue, New York, NY 10001, USA. Tel: +1(212) 584-4662.
License terms

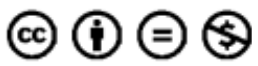



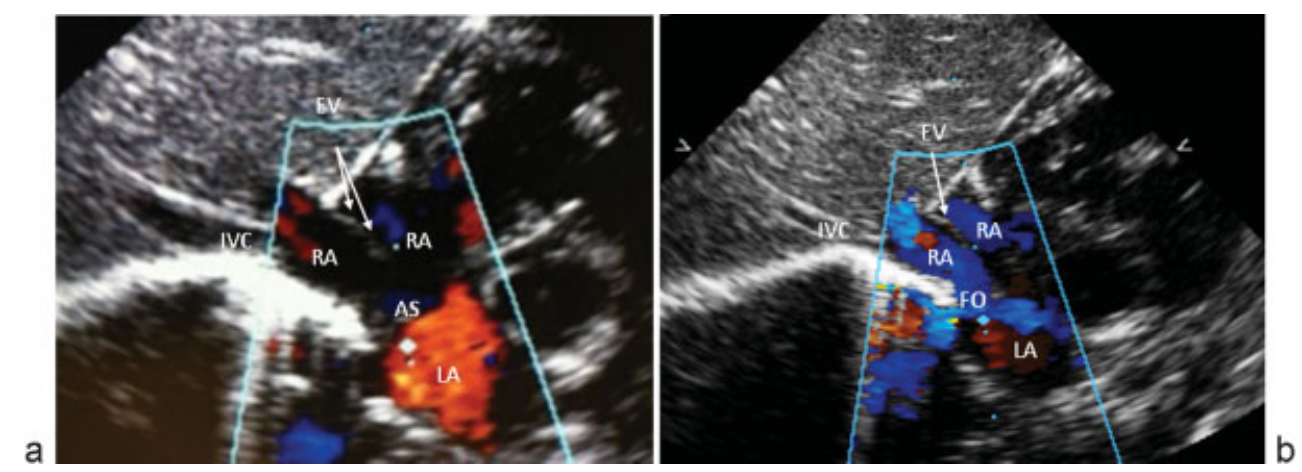

Fig. 1 (a) Subcostal echocardiogram view with color Doppler depicting prominent EV (arrow). (b) Blue color indicates intermittent right-to-left shunting across the FO. AS, atrial septum; EV, Eustachian valve; FO, foramen ovale; IVC, inferior vena cava; LA, left atrium; RA, right atrium.

congenital structural anomalies noted the Echo study. Electrocardiogram (ECG) test was normal for her age. Transnasal fiber optic endoscopy showed a patent right choana, unremarkable nasopharynx, normal vallecula, and base of the tongue, no masses, or cysts, and no signs of laryngomalacia. Barium swallow showed gastroesophageal reflux up to the level of the lower cervical esophagus with no signs of tracheoesophageal fistula or other abnormalities. She remained on 2 L of nasal cannula (NC) for 4 days weaned to room air. It was decided that the patient did not require any medical intervention as she had no respiratory distress or desaturations at rest and had a patent airway. She was sent home with standby $\mathrm{O}_{2}$ and a pulse oximeter with a plan to follow-up in 3 months. At 9 months of age, the patient was found to be doing well, with no further episodes of cyanosis. Repeat Echo showed regression of the EV as well as the PFO with no further intervention required.

\section{Case 2}

A 4-day-old male infant was admitted to the NICU due to multiple failed car seat challenge. The patient was a first twin born at $37^{4 / 7}$ weeks gestation with a birth weight (BW) of $2.25 \mathrm{~kg}$ via repeat C-section with Apgar scores of 9 at both 1 and 5 minutes. All of the mother's antenatal screens were negative, except Group B Streptococcus (GBS) was positive (untreated) with membranes ruptured just before delivery. Due to the baby's small for gestation status, a car seat challenge was performed before discharge. The patient failed three attempts due to desaturations as low as $82 \%$ in addition to a bradycardic event of 96 beats per minute. Abnormal findings lasted for approximately 20 seconds. The patient had otherwise been doing well with normal vital signs at rest and during feeds. The patient was transferred to the NICU where an Echo was performed, which showed a prominent EV, PFO with right-to-left shunting, and good LV function (- Fig. 2). No other anomaly was seen and the coronary sinus ostium appeared normal. ECG was normal for age. The shunting was noted during all phases of the cardiac cycle. No intervention was required at the time and the patient was discharged on the sixth day of life. The patient did not return for scheduled follow-up clinic.

\section{Case 3}

A $3.59 \mathrm{~kg}$ term male infant born at 39 weeks gestation via normal vaginal delivery (NVD) to a 25 year old G1P0 mother; all prenatal screens were negative including GBS. Membranes ruptured 1.5 hours prior to delivery. Apgar scores were 5 and 9 at 1 and 5 minutes, respectively. Due to a maternal history of fever of $101^{\circ} \mathrm{F} 5$ hours prior to delivery, a partial sepsis evaluation was performed, including complete blood count with manual differential, c-reactive protein and blood culture. All results were within normal limits. During a routine nursing evaluation at 45 hours of life, the infant was noted to be dusky colored and febrile with a temperature of $100.6^{\circ} \mathrm{F}$ axillary and a rectal temperature of $101.6^{\circ} \mathrm{F}$. The baby was exclusively breastfed and had lost $9 \%$ of BW. He was transferred to the NICU for antibiotic treatment and hydration rehydration. At around approximately 72 hours of life, the patient started developing multiple episodes of oxygen desaturations to $88 \%$ requiring $2 \mathrm{~L}$ of NC to maintain saturations to greater than $95 \%$. In addition to the desaturations, on initial physical examination, the patient was found to have bilateral hip clunks. A hip ultrasound verified bilateral hip dysplasia. CXR was unremarkable. Echo showed a prominent EV and PFO with right -to-left shunting in diastole and left-to-right-shunting in systole (-Fig. 3). No other anomaly was seen and the coronary sinus ostium appeared normal. ECG test was also normal for age. On day 6 of life, the patient was seen by an Orthopedist who applied a Pavlik harness which was later removed at 2 months of age. After discharge from the NICU, the patient's newborn screen was reported as abnormal for carnitine palmitoyltransferase I deficiency, a fatty acid metabolism disorder. The patient was referred to a regional metabolic center and was tested to be a carrier and only required dietary modification. At the 3 month follow up, the patient was found to be doing well, with normal growth parameters and no further episodes of $\mathrm{O}_{2}$ desaturations. Echo was repeated which showed complete regression of the $\mathrm{EV}$.

\section{Case 4}

A term $3.7 \mathrm{~kg}$ male infant was admitted to the NICU for respiratory distress. He was born via NVD with meconium stained amniotic fluid. All of the mother's antenatal screens 
a
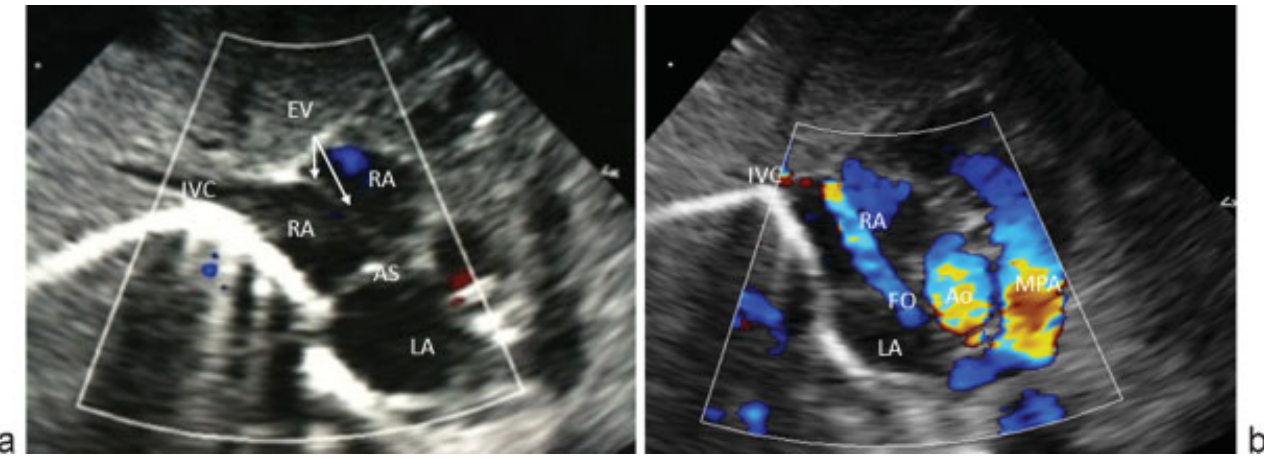

Fig. 2 (a) Subcostal two-dimensional echocardiogram view showing prominent EV (arrow). (b) Color Doppler image showing permanent right-toleft shunting across the FO during the two phases of cardiac cycle (not shown). Ao, aorta; AS, atrial septum; EV, Eustachian valve; FO, foramen ovale; IVC, inferior vena cava; LA, left atrium; MPA, main pulmonary artery; RA, right atrium.
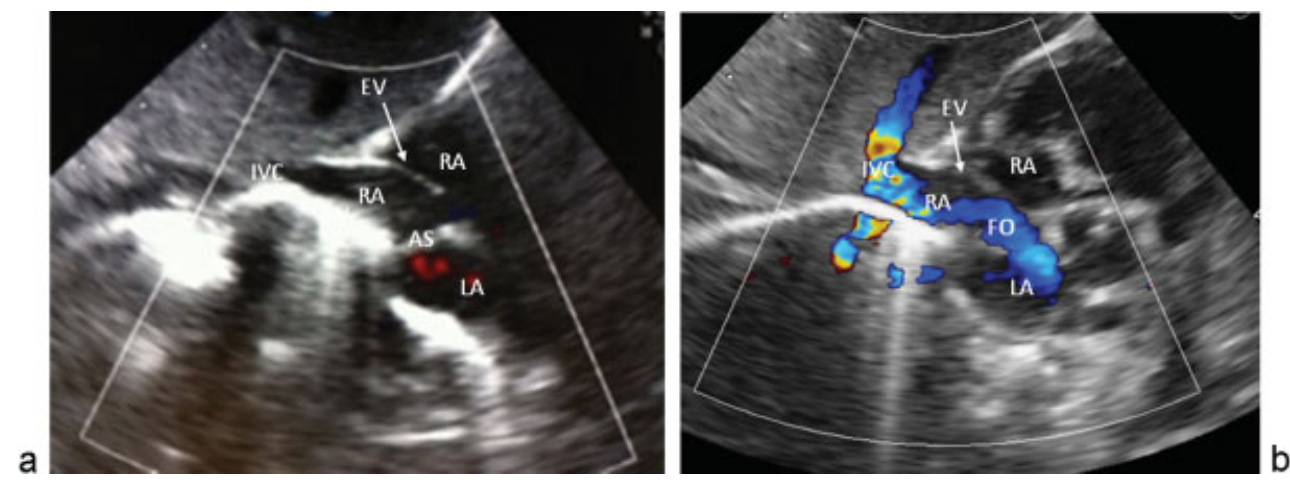

Fig. 3 (a) Subcostal echocardiogram view with color Doppler depicting prominent EV (arrow) with (b) right-to-left shunting across the FO only in diastole. AS, atrial septum; EV, Eustachian valve; FO, foramen ovale; IVC, inferior vena cava; LA, left atrium; RA, right atrium.

were negative, except GBS was positive. Apgar scores were 8 and 8 at both 1 and 5 minutes. CXR was suggestive of meconium aspiration. The patient was started on continuous positive airway pressure (CPAP) and treated with antibiotics. After 2 days, the patient was weaned off CPAP, but had to be placed on NC for desaturations. Lungs were clear on repeat CXR by day 4 of life. The patient continued to require NC to maintain normal $\mathrm{O}_{2}$ saturations, in spite of resolution of other clinical signs of respiratory distress. Echo study showed a prominent EV, PFO with intermittent right-to-left shunting mainly in diastole and good LV function ( - Fig. 4). No other anomaly seen, including $\mathrm{CN}$ and CTD, and the coronary sinus ostium appeared normal. ECG was normal for age. The patient was weaned-off $\mathrm{NC}$ and discharged home on day 9. The patient did not return for scheduled follow-up and repeat Echo.
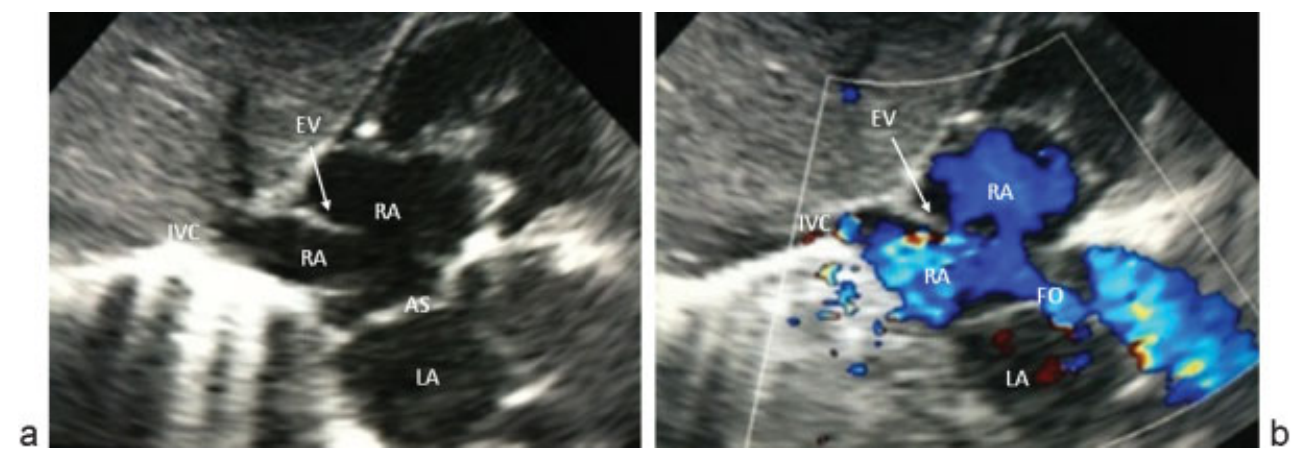

Fig. 4 (a) Subcostal two-dimensional echocardiogram view showing prominent EV (arrow). (b) Color Doppler image showing intermittent rightto-left shunting across the FO only in diastole. AS, atrial septum; EV, Eustachian valve; FO, foramen ovale; IVC, inferior vena cava; LA, left atrium; $R A$, right atrium. 


\section{Discussion}

In this series, we report four cases of intermittent episodes of cyanosis and oxygen desaturation due to a persistent $\mathrm{EV}$, a diagnosis not often considered in patients presenting with these symptoms. Intracardiac right-to-left shunting is believed to be the mechanism of the cyanosis and $\mathrm{O}_{2}$ desaturations in all of our cases. The shunting across the PFO was intermittent in nature and occurred mainly in diastole in three patients, but appeared to be persistent through all phases of the cardiac cycle in only one patient (case 2). Interestingly, three of the infants had other issues which initially confounded the diagnosis. Case 1 presented with stridor which led to an initial suspicion of an airway anomaly. However, the infant's airway was evaluated to be normal and thus her symptoms were attributed to the right-to-left shunting of deoxygenated blood through the EV. In case 4, the baby presented with meconium-stained amniotic fluid, and thus meconium aspiration syndrome was the leading differential diagnosis. The infant's continued need for oxygen despite resolution of other signs of respiratory distress such as tachypnea and accessory muscle use, led to consideration of a cardiac etiology. Finally, although our patient in case 3 had physical findings of bilateral hip dysplasia and was found to be a carrier for the fatty acid metabolism disease, a review of the literature has not shown a correlation between EV remnants and these particular abnormalities. The low 1 minute Apgar score in this baby was unlikely to be related to the $\mathrm{EV}$, as this defect does not lead to significant reduction in perfusion to cause cardiorespiratory depression, particularly in the transitional period immediately following birth.

In all cases, the infants did not require any cardiac intervention. One infant was discharged home with standby $\mathrm{O}_{2}$ but did not require its use. The remainder of the babies were discharged home without oxygen, and remained asymptomatic on follow-up.

A prominent EV may remain after birth, appearing as a thin ridge or a curve-shaped fold of endocardium arising from the anterior rim of the IVC. The lateral horn of this fold tends to adjoin the lower end of the crista terminalis, while at the orifice of the coronary sinus the medial horn joins a semicircular valvular fold known as the Thebesian valve. ${ }^{1}$ In addition, this remnant may even appear as a mobile elongated structure protruding into the right atrial cavity. ${ }^{3}$ The EV has also been described to cross the floor of the right atrium (RA) and insert into the interatrial septum either adjacent to the atrioventricular valves, or higher up, creating an echocardiographic image of a divided RA. ${ }^{3}$ In some cases, it has been known to be large enough to hinder blood flow from the IVC to the RA. Cases have shown that this hindrance in blood flow may result in abnormal intrauterine hemodynamics depriving the right ventricle (RV) of an appropriate blood flow resulting in underdevelopment of the right heart structures. ${ }^{4} \mathrm{~A}$ study by Condoluci et al reported an association with a prominent $\mathrm{EV}$ and congenital heart disease such as tetralogy of Fallot and Ebstein malformation. ${ }^{5}$ In adults, complications such as endocarditis, cerebrovascular events, and pulmonary embolus have been noted but these are thought to be extremely rare events. ${ }^{6-8}$ A report from the Journal of Clinical Pathology 2011 reported a sole case of neonatal EV endocarditis. ${ }^{9}$ In addition, right atrial myxoma and papillary fibroelastoma have also been associated with a prominent EV. ${ }^{10,11}$ Chronic and progressive cyanosis resulting from right-to-left shunting through an atrial septal defect (ASD) as a result of a persistent $\mathrm{EV}$ was described in both a 17 -year-old and a 37-year-old patient. Cardiac catheterization of these patients revealed normal RV and pulmonary arterial pressures. Cyanosis in these two cases was attributed to the EV excluding the IVC from the RA, consequently channeling blood from the IVC directly into the LA. ${ }^{12}$ Intermittent cyanosis during sleep due to prominent EV was reported in a term girl who initially presented with apnea and sinus bradycardia. Right-to-left shunting across the FO was only detected in diastole. ${ }^{13} \mathrm{~A}$ systematic review of the echocardiographic anatomy of the EV can be found by Limacher et al describing the anatomic variations, size, and incidence of the persistent EV in children. ${ }^{14}$ It is extremely important to be able to distinguish a prominent $\mathrm{EV}$ since it can often be mistaken for a prominent $\mathrm{CN}$ or a CTD. $\mathrm{CN}$ is defined as a remnant of the $\mathrm{EV}$ that compromises a membrane of fine fenestrated fibers within the RA. While the EV is usually derived only from the right venous valve, $\mathrm{CN}$ were postulated to be also derived from the embryonic septum spurium, the most prominent anterior pectinate muscle of the crista terminalis. There may also be a continuity of tissue between the Eustachian and Thebesian valves. $\mathrm{CN}$ has been known to result in the persistence of a PFO, formation of an atrial thrombus as well as an atrial septal aneurysm, intense right-to-left shunting, supraventricular arrhythmias, hydrops in early pregnancy, and cyanosis in newborns. ${ }^{15}$ In addition; $\mathrm{CN}$ has been mistaken for pathological structures in the heart such as vegetation, ruptured chordate tendinae, thrombus, and right heart tumors. Although the majority of cases resolve on their own, $\mathrm{CN}$ causing significant clinical findings may need surgical intervention. CTD forms when the entire right sinus venosus valve does not regress and forms a large obstructive flap that separates the RA into two chambers. ${ }^{16} \mathrm{CTD}$ can present with pulmonary valve stenosis or atresia, tricuspid valve abnormalities, and an ASD. These right heart defects primarily stem from a perforated membrane that allows blood flow into the chambers of the RA and through the tricuspid valve into the RV. ${ }^{17}$

\section{Conclusion}

Persistent EV is usually a benign finding, but complications may arise that necessitate surgery, and in rare cases can be fatal. This condition was the etiology of episodic cyanosis and $\mathrm{O}_{2}$ desaturation in our newborn patients. These cases serve as examples to pediatric physicians to not exclude cardiac causes of cyanosis even in the setting of airway or respiratory conditions, and in infants with oxygen desaturations without other signs of respiratory distress. In addition, this remnant must be differentiated from a CN or CTD as these entities 
primarily do not regress and require surgical intervention. Clinicians should also be aware of the variable presentations and natural history of EV to determine the optimal management for their patient.

\section{References}

1 Watson T, Kakar P, Srivastava S, Dhanjal TS. Eustachian valve remnant. Cardiol J 2007;14(5):508-509

2 Sehra R, Ensing G, Hurwitz R. Persistent eustachian valves in infants: course and management in symptomatic patients. Pediatr Cardiol 1998;19(3):221-224

3 Yavuz T, Nazli C, Kinay O, Kutsal A. Giant eustachian valve with echocardiographic appearance of divided right atrium. Tex Heart Inst J 2002;29(4):336-338

4 Schutte DA, Rowland DG, Allen HD, Bharati S. Prominent venous valves in hypoplastic right hearts. Am Heart J 1997;134(3):527-531

5 Condoluci C, di Donato RM, Marcelletti C, Guccione P. Central cyanosis in a newborn infant due to a prominence of the eustachian valve [in Italian]. G Ital Cardiol 1994;24(2):151-154

6 Palakodeti V, Keen WD Jr, Rickman LS, Blanchard DG. Eustachian valve endocarditis: detection with multiplane transesophageal echocardiography. Clin Cardiol 1997;20(6):579-580

7 Kato Y, Fukuoka T, Dembo T, Takeda H, Tanahashi N. Recurrent transient hemiparesis in a patient with a giant persisting Eustachian valve and patent foramen ovale: atypical hemiplegic migraine or paradoxical cerebral embolism? Intern Med 2013; 52(13):1523-1525

8 Maddury J, Alla VM, Misra RC, Maddavapeddi A. Thrombus on the eustachian valve leading to recurrent pulmonary embolism: a rare problem requiring aggressive management. Can J Cardiol 2009; 25(12):e422-e423

9 Chu C, Wallace D, Ofoegbu BN, Hassan I. A case of neonatal eustachian valve endocarditis. J Clin Pathol 2011;64(7): 647-648

10 Teoh KH, Mulji A, Tomlinson CW, Lobo FV. Right atrial myxoma originating from the eustachian valve. Can J Cardiol 1993;9(5): 441-443

11 Arenas Ramírez J, Fernandez Castro C, Otero Chouza M, Duplá Parugues B, Montes Sanchez R. Persistent and redundant eustachian valve simulating atrial tumor: prenatal diagnosis. Ultrasound Obstet Gynecol 2007;29(6):704-707

12 Morishita Y, Yamashita M, Yamada K, Arikawa K, Taira A. Cyanosis in atrial septal defect due to persistent eustachian valve. Ann Thorac Surg 1985;40(6):614-616

13 Yasuda K, Iwashima S, Sugiura H, Ohki S, Seguchi M. Intermittent cyanosis due to prominent eustachian valve in a newborn infant. J Matern Fetal Neonatal Med 2009;22(9):812-815

14 Limacher MC, Gutgesell HP, Vick GW, Cohen MH, Huhta JH. Echocardiographic anatomy of the eustachian valve. Am J Cardiol 1986;57(4):363-365

15 Bendadi F, van Tijn DA, Pistorius L, Freund MW. Chiari's network as a cause of fetal and neonatal pathology. Pediatr Cardiol 2012; 33(1):188-191

16 Aypar E, Sert A, Odabaş D. Unusually prominent Chiari's network prolapsing into the right ventricle in an asymptomatic newborn. Pediatr Cardiol 2013;34(4):1017-1019

17 Martínez-Quintana E, Rodríguez-González F, Marrero-Santiago H, Santana-Montesdeoca J, López-Gude MJ. Cor triatriatum dexter versus prominent Eustachian valve in an adult congenital heart disease patient. Congenit Heart Dis 2013;8(6):589-591 